\title{
An Analysis of Inconsistent and Incomplete Necker Cubes
}

\author{
Chris Mortensen \\ Departments of Philosophy \\ Universities of Adelaide and Melbourne \\ Chris.Mortensen@adelaide.edu.au
}

Received by Greg Restall

Published March 30, 2006

http://www .philosophy. unimelb.edu.au/ajl/2006

(c) 2006 Chris Mortensen

\begin{abstract}
This paper aims to distinguish and classify sixteen versions of the Necker cube. In particular, it is shown how to describe inconsistent and incomplete theories which correspond in a systematic way to these sixteen diagrams. Concerning two of these sixteen cubes, there is a natural intuition that there is a sense in which they inconsistent. It is seen that this intuition is vindicated by an analysis in which their corresponding theories turn out to be globally inconsistent but not locally inconsistent, while various other cubes of the sixteen are merely locally inconsistent. The Routley functor is seen to be useful in classifying the relations between these diagrams.
\end{abstract}

\section{INTRODUCTION}

This paper aims to distinguish and classify sixteen versions of the Necker cube. In particular, it is shown how to describe inconsistent and incomplete theories which correspond in a systematic way to these sixteen diagrams. Concerning two of these sixteen cubes, there is a natural intuition that there is a sense in which they inconsistent. It is seen that this intuition is vindicated by an analysis in which their corresponding theories turn out to be globally inconsistent but not locally inconsistent, while various other cubes of the sixteen are merely locally inconsistent. The project falls into the general area of the application of inconsistent mathematics to geometry, and conversely the emergence of inconsistent theories from geometric figures, as described for example in Mortensen (2002). The project should be distinguished from, though related to, modeling logics in geometry, as exemplified for example in Shin and Lemon (2003), and the literature referenced therein. To explain these opening remarks more fully, it is necessary to make some preliminary distinctions. 
We begin with the usual Necker "cube". I place this initially in scare quotes for reasons which become apparent as we proceed, namely that it is better called a Necker cube diagram.

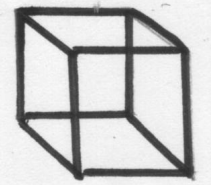

DIAGRAM I: The usual Necker

The popular story of the Necker cube is that it is ambiguous, namely that perceiver's gestalt switches between two aspects, first with one face in front, then the other. This story will be altered and extended as we proceed. The two ways to "disambiguate" the Necker cube can be displayed by introducing occlusions at the two internal crossings, in the manner of the convention governing knot diagrams in knot theory.
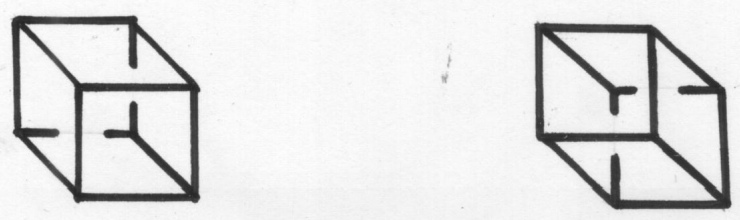

DIAGRAM 2: Two disambiguated Neckers with occlusions

This can be made even more apparent by bringing in colours as well: red lines for one face, blue lines for its opposite face, and green for connecting lines.
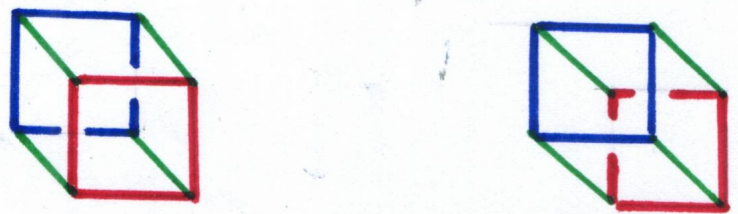

DIAGRAM 3: Disambiguated Neckers with occlusions and colour

But now notice something important: the devices of occlusion and colour provide for two "impossible" cubes, where one of the two crossings favours the red, while the other crossing favours the blue. We note that this effect is more marked with animation
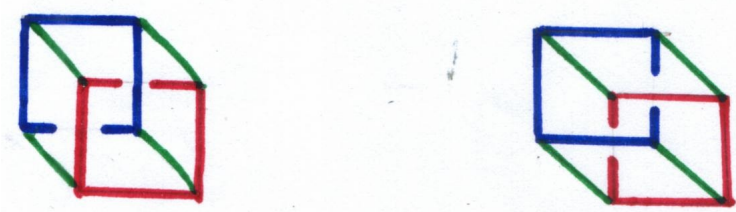

DIAGRAM 4: Impossible Neckers

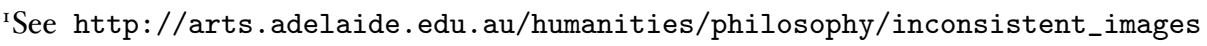
for examples. 
This paper aims to explain the experience of the two impossible cubes by defining an inconsistent theory which describes the content of the experience. It is a first step in the more general cognitive project (Mortensen 2002) of explaining the experiences of impossible images such as those by Reutersvaard and Escher, in terms of internalised inconsistent geometrical theories. The sense of impossibility is explained as conflicting visual experiences and expectations, and the conflict is explained in terms of inconsistency between contents.

\section{LEVEL I ANALYSIS: TWO DIMENSIONS}

We begin with the characteristics of the flat image, namely the $2 \mathrm{D}$ collection of lines and points at which they intersect or cross. This amounts to all you can see without reading a third dimension into your perceptual construct. There are 8 points and $\mathrm{I} 2$ lines. In saying that there are $\mathrm{I} 2$ lines, I am including as a single line of the same colour those lines which are broken in the diagrams with crossings. This way of speaking leads to some descriptive simplicity when we move up a dimension to $3 \mathrm{D}$.

We begin to present a set of axiomatic theories. The background logic is left undetermined, but is thought of as paraconsistent and paracomplete, such as a suitable 4-valued logic which sustains non-trivial inconsistent and incomplete theories. Perhaps the simplest suitable background logic would be Anderson/Belnap first degree entailment F DE (see e.g. Anderson and Belnap ${ }_{975} \mathrm{Ch}_{3}$, for a simple 4-valued semantics see Priest 20OI Ch8). FDE is the constant first degree part of many natural logics, and satisfies the conditions for the Routley Functor to be applicable (see below, Section 3). As will become apparent, the interesting facts about these Necker cubes and their classifications are all at the zero degree level of atomic relations compounded with $(\wedge, \vee, \sim)$, together with entailments $\rightarrow$ between them. Quantifiers $(\exists, \forall)$ can be eliminated in favour of disjunctions and conjunctions because these geometrical objects contain only a finite number of elements (finite numbers of vertices, edges, faces and crossings). In particular, higher-degree nestings of entailments or implications, do not seem to reflect themselves in the pictures. Logicians' disputes over the properties of nested entailments are thus not relevant here. This is in line with the observation that inconsistent mathematics is invariant over a large class of background logics. On the other hand, it is worth noting in passing that not all paraconsistent techniques are equally suitable. For example, non-adjunctive techniques do not seem to be applicable (though they may have application for other styles of inconsistent pictures, such as the Schuster fork).

We focus on the two crossings as the most important part of the diagram, since it is by changing what is happening there that enables a switch from inconsistent to consistent diagrams, and back again. Fix and describe the whole diagram but for the two crossings. Call the crossings $C_{1}$ and $C_{2}$. At each crossing can be placed a red point RP, a blue point BP, both or neither. For the coloured diagrams, this can be likened to dropping paint of the appropriate 
colour at the crossings. The basic relation is $x @ C$, where $x$ is a coloured point and $C$ is a crossing. Thus for crossing $C_{1}$, we have four possible theories:

(Ax.I) RP@ $\mathrm{C}_{1}$ (but lacking BP@ $\mathrm{C}_{1}$ )

(Ax.2) BP@ $\mathrm{C}_{1}$ (but lacking RP@ $\mathrm{C}_{1}$ )

(Ax.3) Both RP@ $\mathrm{C}_{1}$ and $\mathrm{BP} @ \mathrm{C}_{1}$

(Ax.4) Neither RP@C1 nor BP@ $\mathrm{C}_{1}$

Similarly for $\mathrm{C}_{2}$. Thus there are $\mathrm{I} 6$ possible Necker diagrams that can be built out of these elements.
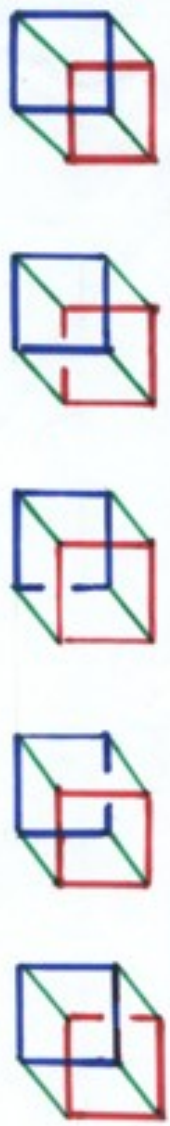
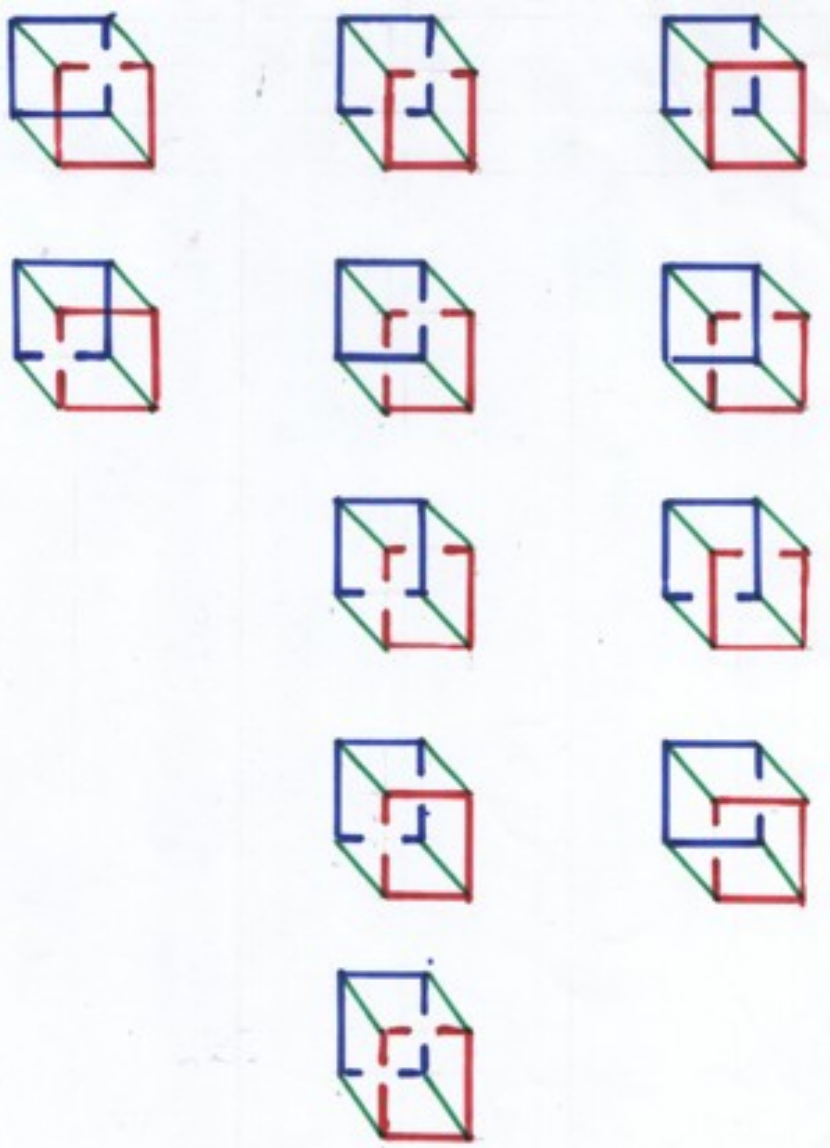

Diagram 5: Sixteen Necker cubes

What we have with the sixteen Neckers above, is a generalization of the theory of knots in which only crossings of the first two types occur. Now it might be thought to be problematic that there can be both red and blue at $C_{1}$ at once. After all, this might seem to violate the natural principle that a thing cannot be both red and blue all over at the same time. However, at the present level of 
analysis, there is nothing obviously inconsistent about placing both red paint and blue paint at $C_{1}$ at the same time. Various things might ensue, so no assumptions should be made as to extra-logical principles.

Having said that, I wish to signal endorsement of this natural principle. This can be included as an axiom of local consistency of the @ relation:

$(A x . L o C o n @)(\forall C)(R P @ C \rightarrow \sim B P @ C)$

This axiom is routinely satisfied by Ax.I, Ax.2, and Ax.4. It is apparent that in the presence of Ax.3, an inconsistent theory is generated. These facts can be expressed using some definitions:

Definition I (LOCAL CONSISTENCy of @) A theory Th is locally consistent at C with respect to @ iff for each colour $x$ not both $x @ C$ and $\sim x @ C$ are in Th. A theory Th is locally consistent with respect to @ iff it is locally consistent for all C with respect to @.

DEFINITION 2 (LOCAL COMPLETENESS OF @) A theory Th is locally complete at $\mathrm{C}$ with respect to @ iff for each colour $x$ either $x @ C$ or $\sim x @ C$ are in Th. A theory Th is locally complete with respect to @ iff it is locally complete for all C with respect to @.

(One often drops the “@” when it is obvious which relation is being discussed.) Thus, Ax.I and Ax.2 correspond to locally consistent and complete theories, Ax.3 to a locally inconsistent theory and Ax.4 to a locally incomplete theory. A point to be noted here is that Ax.I, 2, 4 are not quite "axioms" in the traditional sense, since they involve exclusions as well. But that is no real problem, one can either employ an exclusion operator in the metalanguage, or simply take Ax.I-4 not as axioms, but as more general stipulations of the theory of interest.

In terms of the sixteen Neckers in Diagram 5, it can be verified that:

I. The theories of the Neckers in the first column are locally inconsistent and complete. In particular, the top left diagram (the traditional Necker) is trivial, in that all atomic sentences of the form P@C hold, that is Ax.3 holds for both crossings. All others have one inconsistent and one consistent and complete crossing, that is Ax.3 holds for one crossing, and either Ax.I or Ax.2 holds for the other.

2. Those in the second column are locally inconsistent and incomplete. That is, Ax.3 holds for one crossing and Ax.4 for the other.

3. Those in the third column are locally consistent and incomplete. That is, Ax.4 holds for one or both crossings, and Ax.I or Ax.2 holds where Ax.4 does not. The bottom diagram of this column has Ax.4 holding at both crossings; that is the theory of this diagram is incomplete at both crossings, thus has no non-logical sentences $\mathrm{P} @ \mathrm{C}$ or $\sim \mathrm{P} @ \mathrm{C}$ holding. All 
others in the third column are consistent and incomplete at one crossing, and consistent and complete at the other.

4. Those in the fourth column are all locally consistent and complete. The top two are the classical cases displayed also in Diagram 3, where Ax.I holds at both crossings, or Ax.2 does. The bottom two are the impossible Neckers of Diagram 4. As can be seen, Ax.r holds at one crossing, and Ax.2 at the other.

One can think of the Necker diagrams above as "representations" of such ocally inconsistent and incomplete theories, an extension of the convention of having a broken line represent a locally consistent and complete situation as with knot theory.

A convergent motivation is that the evident duality between Ax. 3 and Ax.4 can be given a pleasing theoretical symmetry. As we see in the next section, this theoretical symmetry can be described with the Routley Functor (the Routley $*$, which contributes to the theoretical usefulness of that notion.

This section is concluded by observing that the two impossible Neckers of Diagram 4 have not yet been given an explanation in terms of an inconsistent theory, though they have been set into an appropriate preliminary framework for doing so. So far, they are locally consistent and complete. Only if an inconsistent theory is forthcoming, can it be said that the sense that we have here of figures that are somehow contradictory, has been justified. The remainder of this paper is devoted to this task

\section{LEVEL 2 ANALYSIS: THREE DIMENSIONS - VERTICES AND EDGES}

We move to what $3 \mathrm{D}$ objects these $2 \mathrm{D}$ diagrams represent. That is, we are (mentally) constructing a $3 \mathrm{D}$ object out of $2 \mathrm{D}$ data, in the usual way of depth perception (one eye). To mark the difference, we introduce the usual geometrical terminology of vertices, edges and faces. Thus, all 8 points become 8 vertices, and all of $\mathrm{I} 2$ lines become I 2 edges. At the present level we have only vertices and edges, not faces which come at a later level.

Crossings are seen as a 2D artefact of viewing a 3D object from a particular point of view, that is as devices for registering which of two points in $3 \mathrm{D}$ space that project to the same point in 2D is closer, and which is further away. Again, this is in line with the corresponding treatment of $2 \mathrm{D}$ knot diagrams as projections of knots in 3-space. However, it is important to see the present project as building up a 3D object from $2 \mathrm{D}$ data. So we want to preserve the viewing aspect of the picture. Thus the main relation we want is that of occlusion, written " $E_{1}$ occ $E_{2}$ ". This is a relation between edges $E_{1}$ and $E_{2}$. In these theories, there are just four edges involved in the occlusion, two red and two blue.

Hence, where RE is a red edge and $B E$ is a blue edge, we may define: 
DEFINITION 3 Occlusion

$$
\begin{aligned}
& R E \text { occ } B E={ }_{d f}(\exists C)(R E \text { and } B E \text { cross at } C \text { and } R P @ C) \\
& B E \text { occ } R E={ }_{d f}(\exists C)(R E \text { and } B E \text { cross at } C \text { and } B P @ C)
\end{aligned}
$$

Thus, while the occlusion relation holds between 3D edges, it derives from data in the 2D diagrams we began with.

It is clear that where Ax.I or Ax.2 hold for each crossover, this ensures that, for each crossover, just one of the above two conditions hold (again as in knot theory), since we do not have both RP@C and BP@C for any crossover C. When Ax.3 holds at a crossover, both RE occ BE and BE occ RE hold there. When Ax.4 holds at C, neither RE occ BE nor BE occ RE hold there. These can then be read back into the diagrams

It then becomes of interest to consider a local consistency axiom for occ. It says that if one edge occludes another then the latter does not occlude the former, i. e. the asymmetry of occ. In knot theory this is so standard that it is rarely mentioned.

(Ax.LoConOcc) $(\forall x)(\forall y)((x$ occ $y) \rightarrow \sim(y$ occ $x))$

This axiom expresses the consistent state of affairs that holds when one edge occludes another at their crossing. Again, it is to be endorsed and included here. For diagrams where there is just one colour at the crossing point between the two edges, LoConOcc is satisfied, as it is if Ax.4 holds. When Ax.3 holds there is a theory which is locally inconsistent with respect to the occ relation. That is, LoConOcc is satisfied if we treat a crossing where both colours are present as inconsistent, since the presence of both RE occ BE and BE occ RE ensures also $\sim(R E$ occ $B E)$ and $\sim(B E$ occ RE). Summing up:

THEOREM I If LoConOcc is added to the Axioms I-4 above, there are the following consequences: For Ax.I and 2, the occlusion-theory is both consistent and complete. For Ax.3, the occlusion-theory is locally inconsistent and complete. For Ax.4, the occlusiontheory is locally consistent and incomplete

It is further seen that the relations between these four axioms can be described using the Routley Functor $*$.

Definition $4(\operatorname{ROUTLEY} *)$ If $T h$ is a theory, then $\operatorname{Th}^{*}$ is $\{A: \sim A \notin T h\}$.

It is known (Routley and Routley 1972) that, for a broad class of background logics (including FDE) satisfying only weak constraints, (I) $\mathrm{Th}^{*}$ is deductively closed iff $T h$ is, (2) $\mathrm{Th}^{* *}=\mathrm{Th},(3){ }^{*}$ snips out pairs $(\mathrm{A}, \sim \mathrm{A})$ from $\mathrm{Th}$, where Th is inconsistent, and adds pairs $(A, \sim A)$ where Th is incomplete, so (4) $\mathrm{Th}^{*}$ is inconsistent iff Th is incomplete, $(5) \mathrm{Th}^{*}$ is incomplete iff Th is inconsistent, and (6) $\mathrm{Th}^{*}=\mathrm{Th}$ iff $\mathrm{Th}$ consistent and complete.

If we then write '(Ax.I)' for the zero degree (@, occ) theory generated from Ax.I, LoCon@ and LoConOcc, we have: 
TheOREM 2 We have $(\mathrm{Ax} . \mathrm{I})^{*}=(\mathrm{Ax} . \mathrm{I}),(\mathrm{Ax} .2)^{*}=(\mathrm{Ax} .2),(\mathrm{Ax} .3)^{*}=(\mathrm{Ax} .4)$ and $(\mathrm{Ax} .4)^{*}=(\mathrm{Ax} \cdot 3)$

Proof: First, (Ax.I) is consistent and complete, as we have seen. Hence by (4) of the previous paragraph, (Ax.I) ${ }^{*}=$ (Ax.I). Second, similarly for (Ax.2). Third, we have seen that if $A x .3$ holds, all of RP@C, $\sim R P @ C, B P @ C, \sim B P @ C$, $R E$ occ $B E, \sim(R E$ occ $B E), B E$ occ $R E$, and $\sim(B E$ occ $R E)$ are included in the theory. Since the effect of the Routley $*$ is to remove inconsistent pairs $(A, \sim A)$, in $(\mathrm{Ax} .3)^{*}$, all of the above atomic sentences are removed. But that is exactly the condition of (Ax.4). Fourth, similarly for (Ax.4), since the effect of $*$ on missing pairs $(A, \sim A)$ is to replace both of them.

To sum up, we can now say that the 16 Necker diagrams above are classifiable as: 5 locally inconsistent and locally complete 2 locally inconsistent and locally incomplete 5 locally consistent and incomplete. 4 locally consistent and locally complete. These four classes are the four columns of Diagram 5 respectively. Moreover, the elements of the first class and are $*$-duals of the third class, the elements of the second class are $*$-duals of each other, and the elements of the fourth class are self- $*$-dual.

It is the last class of four that we are interested in. It contains the two "normal" Neckers and the two impossible Neckers we began with (Diagrams 2 and 3), which remain the target of our analysis. Though the latter two are impossible, they are (locally) consistent and complete. How can this be? It requires an analysis that demonstrates an inconsistency somewhere, but where? The answer lies in inconsistency in some more global sense. The problem that was set in the beginning, it will be recalled, is to give an inconsistent theory which would capture these essentially $3 \mathrm{D}$ mental constructions.

\section{LEVEL 3 ANALYSIS: THREE DIMENSIONS - FACES}

We proceed to add faces to the theory. A face (for the cube) is a figure having four edges intersecting in four vertices. The usual consistent theory is that cubes have six faces. Using colours for the edges, we can see that in the consistent case there is there is one face all of whose edges are red, and one face all of whose edges are blue, while the other four faces have at least one green edge. There are no occlusions involving green edges, so we focus just on the red and blue faces, RF and BF.

In parallel with the addition of edges and occlusions in the previous section, we define the relation "in front of" for faces. This relation obtains only between the red and the blue faces. Note that we do not describe this relation as a direct extension of the occlusion relation between edges, if only because it is not appropriate for Necker cubes, whose faces are not opaque: only the edges occlude. Taking the lead from the consistent case, we define: 
DEFINITION 5 in front of

$F_{1}$ in front of $F_{2}=$ df some edge of $F_{1}$ occludes some edge of $F_{2}$

In case of the "normal" Neckers (Diagrams 2 and 3) we have that RF is in front of $\mathrm{BF}$, or vice versa, but not both.

It might be thought that we should have required that all edges of $\mathrm{F} 1$ occlude all edges of F2. However this is unnecessary in the normal consistent and complete cases. Independently, if one tries out the cognitive experiment of covering one of the crossovers and asking which colour occludes which at that crossing, the natural answer to give is that it is the same as the visible crossing (I owe this observation to Lloyd Humberstone). Thus, the definition is built into our expectations.

Now it is appropriate to consider an axiom of global consistency for the relation 'in front of'.

$($ Ax.GlobCon) $(\forall x)(\forall y)((x$ in front of $y) \rightarrow \sim(y$ in front of $x))$

This asymmetry of in front of guarantees, for the two problematic Neckers (Diag.4), that we have RF in front of BF, $\sim(R F$ in front of $B F), B F$ in front of $R F$ and $\sim(B F$ in front of $R F)$. Introducing a definition:

Definition 6 (GLObal InCONSISTENCy) A theory is globally inconsistent iff it is inconsistent with respect to the relation 'in front of. It is otherwise globally consistent.

Then it follows that the two problematic Neckers are locally consistent and complete but globally inconsistent. I submit that this would seem to be independently reasonable as an expression of our intuition that the globally anomalous Neckers we began with are inconsistent. They are "defective in facemaking" because some faces end up being inconsistently in front of each other.

Thus, the promised theories for the whole class of sixteen Neckers are one of Ax.I-4 for each crossing, plus the two local consistency axioms, LoCon@ and LoConOcc, plus GlobCon. The three latter axioms sometimes have no effect (when Ax.I, 2 or 4 hold), otherwise render the theory inconsistent.

\section{CONCLUSION}

Overall, we have seen that these axioms and definitions combine to produce varying local and global inconsistencies, consistencies, incompletenesses and completenesses. We saw that the Routley Functor is useful in describing and unifying these properties. In particular, the intuition that the two anomalous Neckers we identified at the beginning are globally inconsistent in cognitive content, is given a precise explication. 


\section{REFERENCES}

Anderson, A. R., and Belnap Jr., N. D. (1975) Entailment, Volume I, Princeton, Princeton University Press.

Mortensen, C. (2002), "Towards a Mathematics of Impossible Pictures" in W. Carnielli, M. E. Coniglio and Itala M. L. D'Ottaviano editors, Paraconsistency, the Logical Way to the Inconsistent, New York, Marcel Dekker, 445-454.

Priest, G. (200I), An Introduction to Non-Classical Logic, Cambridge, Cambridge University Press.

Routley, R. and Routley, V. (1972), “The Semantics of First Degree Entailment”, Nô̂s $6,335-359$.

Shin, S.-J., and Lemon, O., (2003) "Diagrams" Stanford Encyclopedia of Philosophy (Winter 2003 edition), http://plato.stanford.edu/entries/diagrams 
The Australasian fournal of Logic (ISSN I448-5052) disseminates articles that significantly advance the study of logic, in its mathematical, philosophical or computational guises. The scope of the journal includes all areas of logic, both pure and applied to topics in philosophy, mathematics, computation, linguistics and the other sciences.

Articles appearing in the journal have been carefully and critically refereed under the responsibility of members of the Editorial Board. Only papers judged to be both significant and excellent are accepted for publication.

The journal is freely available at the journal website at

http://www .philosophy . unimelb.edu.au/ajl/

All issues of the journal are archived electronically at the journal website.

SUBSCRIPTIONS Individuals may subscribe to the journal by sending an email, including a full name, an institutional affiliation and an email address to the managing editor at ajl-editors@unimelb.edu.au Subscribers will receive email abstracts of accepted papers to an address of their choice. For institutional subscription, please email the managing editor at ajl-editors@unimelb.edu.au.

Complete published papers may be downloaded at the journal's website at http://www.philosophy.unimelb.edu.au/ajl/ The journal currently publishes in pdf format.

Submission The journal accepts submissions of papers electronically. To submit an article for publication, send the ${ }^{\Delta} \mathrm{T}_{\mathrm{E}} \mathrm{X}$ source of a submission to a member of the editorial board. For a current list of the editorial board, consult the website.

The copyright of each article remains with the author or authors of that article. 